\title{
Solution of Laplace's Differential Equation and Fractional Differential Equation of That Type
}

\author{
Tohru Morita $^{1}$, Ken-ichi Sato ${ }^{2}$ \\ ${ }^{1}$ Tohoku University, Sendai, Japan \\ ${ }^{2}$ College of Engineering, Nihon University, Koriyama, Japan \\ Email: senmm@jcom.home.ne.jp
}

Received August 19, 2013; revised September 19, 2013; accepted September 26, 2013

Copyright (C) 2013 Tohru Morita, Ken-ichi Sato. This is an open access article distributed under the Creative Commons Attribution License, which permits unrestricted use, distribution, and reproduction in any medium, provided the original work is properly cited.

\begin{abstract}
In a preceding paper, we discussed the solution of Laplace's differential equation by using operational calculus in the framework of distribution theory. We there studied the solution of that differential equation with an inhomogeneous term, and also a fractional differential equation of the type of Laplace's differential equation. We there considered derivatives of a function $u(t)$ on $(0, \infty)$, when $u(t)$ is locally integrable on $(0, \infty)$, and the integral $\int_{0}^{1} u(t) \mathrm{d} t$ converges. We now discard the last condition that $\int_{0}^{1} u(t) \mathrm{d} t$ should converge, and discuss the same problem. In Appendices, polynomial form of particular solutions are given for the differential equations studied and Hermite's differential equation with special inhomogeneous terms.

Keywords: Laplace's Differential Equation; Kummer's Differential Equation; Fractional Differential Equation; Distribution Theory; Operational Calculus; Inhomogeneous Equation; Polynomial Solution
\end{abstract}

\section{Introduction}

Yosida [1,2] discussed the solution of Laplace's differential equation (DE), which is a linear DE, with coefficients which are linear functions of the variable. The DE which he takes up is

$$
\begin{aligned}
& \left(a_{2} t+b_{2}\right) y^{\prime \prime}(t)+\left(a_{1} t+b_{1}\right) y^{\prime}(t) \\
& +\left(a_{0} t+b_{0}\right) y(t)=0, \quad t>0,
\end{aligned}
$$

where $a_{l}$ and $b_{l}$ for $l=0,1,2$ are constants. His discussion is based on Mikusiński's operational calculus [3]. Yosida $[1,2]$ gave there only one of the solutions of the DE (1.1).

In the preceding paper [4], we discussed the solution of an fractional differential equation (fDE) of the type of $\mathrm{DE}(1.1)$, that is given by

$$
\begin{aligned}
& \left(a_{2} t+b_{2}\right){ }_{0} D_{R}^{2 \sigma} u(t)+\left(a_{1} t+b_{1}\right){ }_{0} D_{R}^{\sigma} u(t) \\
& +\left(a_{0} t+b_{0}\right) u(t)=f(t), \quad t>0,
\end{aligned}
$$

for $\sigma=1$ and $\sigma=1 / 2$. Here ${ }_{0} D_{R}^{\sigma} u(t)$ for $\sigma \in \mathbb{R}_{>0}$ is the Riemann-Liouville fractional derivative (fD) defined in Section 2. We use $\mathbb{R}$ to denote the set of all real numbers, and $\mathbb{R}_{>b}:=\{x \in \mathbb{R} \mid x>b\}$. When $\sigma$ is equal to an integer $n \in \mathbb{Z}_{>0},{ }_{0} D_{R}^{n} u(t)=\frac{\mathrm{d}^{n}}{\mathrm{~d}^{n}} u(t)$. When $\sigma=1,(1.2)$ is the inhomogeneous DE for (1.1). We use $\mathbb{Z}$ to denote the set of all integers, and $\mathbb{Z}_{>a}:=\{n \in \mathbb{Z} \mid n>a\}, \mathbb{Z}_{<b}:=\{n \in \mathbb{Z} \mid n<b\}$ and $\mathbb{Z}_{[a, b]}:=\{n \in \mathbb{Z} \mid a \leq n \leq b\}$ for $a, b \in \mathbb{Z}$ satisfying $a<b$. We use $\lceil x\rceil$ for $x \in \mathbb{R}$, to denote the least integer that is not less than $x$.

In [4], we adopt operational calculus in the framework of distribution theory developed for the solution of the fDE with constant coefficients in $[5,6]$. In [4], we give the recipe of obtaining the solution of the inhomogeneous equation as well as the homogeneous one, and we show how the set of two solutions of the homogeneous equation is attained.

In [4], we adopt the usual definition of the Riemann-Liouville $\mathrm{fD}$, which defines ${ }_{0} D_{R}^{\beta} f(t)$ only for such a locally integrable function $f(t)$ on $\mathbb{R}_{>0}$ that $\int_{0}^{1} f(t) \mathrm{d} t$ is finite. Practically, we adopt Condition B in [4], which is 
Condition IB $u(t) H(t)$ and $f(t) H(t)$ are expressed as a linear combination of $g_{v}(t)$ for $v>0$.

Here $H(t)$ is Heaviside's step function, and when $f(t)$ is defined on $\mathbb{R}_{>b}, f(t) H(t-b)$ is assumed to be equal to $f(t)$ when $t>b$ and to 0 when $t \leq b$. $g_{v}(t)$ is defined by

$$
g_{v}(t)=\frac{1}{\Gamma(v)} t^{\nu-1},
$$

for $v \in \mathbb{R}_{>0}$, where $\Gamma(v)$ is the gamma function.

In [4], we take up Kummer's DE as an example, which is

$$
t \cdot \frac{\mathrm{d}^{2}}{\mathrm{~d} t^{2}} u(t)+(c-t) \cdot \frac{\mathrm{d}}{\mathrm{d} t} u(t)-a \cdot u(t)=0, t>0,
$$

where $c, a \in \mathbb{R}$ are constants. If $c \notin \mathbb{Z}$, one of the solutions given in $[7,8]$ is

$$
{ }_{1} F_{1}(a ; c ; t):=\sum_{n=0}^{\infty} \frac{(a)_{n}}{(c)_{n} n !} t^{n},
$$

where $(a)_{n}=\prod_{k=0}^{n-1}(a+k)$ for $a \in \mathbb{R}$ and $n \in \mathbb{Z}_{>0}$, and $(a)_{0}=1$. The other solution is

$$
t^{1-c} \cdot{ }_{1} F_{1}(a-c+1 ; 2-c ; t) \text {. }
$$

In [4], if $c<2$, we obtain both of the solutions. But when $c \geq 2,(1.6)$ does not satisfy Condition IB and we could not get it.

In a recent review [9], we discussed the analytic continuations of $\mathrm{fD}$, where an analytic continuation of Riemann-Liouville $\mathrm{fD},{ }_{0} D_{R}^{\beta} f(t)$, is such that the $\mathrm{fD}$ exists even for such a locally integrable function $f(t)$ on $\mathbb{R}_{>0}$ that $\int_{0}^{1} f(t) \mathrm{d} t$ diverges. In the present paper, we adopt this analytic continuation of ${ }_{0} D_{R}^{\beta} f(t)$.

In place of the above Condition IB, we now adopt the following condition.

Condition A $u(t) H(t)$ and $f(t) H(t)$ are expressed as a linear combination of $g_{v}(t) H(t)$ for $v \in S$, where $S$ is a set of $v \in \mathbb{R}_{>-M} \backslash \mathbb{Z}_{<1}$ for some $M \in \mathbb{Z}_{>-1}$.

As a consequence, we can now achieve ordinary solutions for (1.2) of $\sigma=1$. For (1.4), we obtain both solutions (1.5) and (1.6) if $c \notin \mathbb{Z}$.

It is the purpose this paper to show how the presentation in [4] should be revised, with the change of definition of $\mathrm{fD}$ and the replacement of Condition IB with Condition A.

In Section 2, we prepare the definition of RiemannLiouville $\mathrm{fD}$ and then explain how the function $u(t)$ and its $\mathrm{fD}$ in (1.2) are converted into the corresponding distribution $\tilde{u}(t)$ and its $\mathrm{fD}$ in distribution theory, and also how $\tilde{u}(t)$ is converted back into $u(t)$. After these preparation, a recipe is given to be used in solving the
fDE (1.2) with the aid of operational culculus in Section 3. In this recipe, the solution is obtained only when $a_{2} \neq 0$ and $b_{2}=0$. When $\sigma=\frac{1}{2}, b_{1}=0$ is also required. An explanation of this fact is given in Appendices C and D of [4]. In Section 4, we apply the recipe to (1.2) where $\sigma=1$ and $a_{0}=0$, of which special one is Kummer's DE. This is an example which Yosida $[1,2]$ takes up. In Section 5, we apply the recipe to the fDE with $\sigma=\frac{1}{2}$, assuming $a_{0}=0$.

For the Hermite DE with inhomogeneous term, Levine and Malek [10] showed that there exist particular solutions in the form of polynomial. In Appendices $\mathbf{A}$ and $\mathbf{C}$, we show that such a solution exists for the DE and fDE studied in Sections 4 and 5, respectively. In Appendix B, we show how the results presented in [10] are derived from those in Appendix A.

\section{Formulas}

We now adopt Condition A. We then express $u(t) H(t)$ as follows;

$$
u(t) H(t)=\sum_{v \in S} u_{v-1} g_{v}(t) H(t),
$$

where $u_{v-1} \in \mathbb{R}$ are constants.

Lemma 1 For $v \in \mathbb{R} \backslash \mathbb{Z}_{<1}$,

$$
t \cdot g_{v}(t)=v \cdot g_{v+1}(t)
$$

Proof By (1.3), for $v \notin \mathbb{Z}_{<1}$, we have

$$
t \cdot g_{v}(t)=\frac{1}{\Gamma(v)} t^{v}=\frac{v}{\Gamma(v+1)} t^{v}=v \cdot g_{v+1}(t)
$$

\subsection{Riemann-Liouville Fractional Integral and Derivative}

Let $f(t) H(t-b)$ be locally integrable on $\mathbb{R}$. We then define the Riemann-Liouville fractional integral, ${ }_{b} \tilde{D}_{R}^{-\lambda} f(t)$, of order $\lambda \in \mathbb{R}_{>0}$ by

$$
{ }_{b} \tilde{D}_{R}^{-\lambda} f(t)=\frac{1}{\Gamma(\lambda)} \int_{b}^{t}(t-x)^{\lambda-1} f(x) \mathrm{d} x, t>b .
$$

We then define the Riemann-Liouville $\mathrm{fD},{ }_{b} D_{R}^{\beta} u(t)$, of order $\beta \in \mathbb{R}$, by

$$
{ }_{b} D_{R}^{\beta} u(t)=\frac{\mathrm{d}^{N}}{\mathrm{~d} t^{N}}\left[{ }_{b} \tilde{D}_{R}^{\beta-N} u(t)\right], t>b,
$$

if it exists, where $N=\max \{\lceil\beta\rceil, 0\}$, and ${ }_{b} \tilde{D}_{R}^{0} u(t)=u(t)$ for $t>b$.

For $v>0$, we have

$$
{ }_{0} D_{R}^{\beta} g_{v}(t)= \begin{cases}g_{v-\beta}(t), & v-\beta \in \mathbb{R} \backslash \mathbb{Z}_{<1}, \\ 0, & v-\beta \in \mathbb{Z}_{<1} .\end{cases}
$$


If we assume that $\beta=-\lambda$ takes a complex value, ${ }_{0} \tilde{D}_{R}^{\beta} g_{v}(t)$ by definition (2.3) is analytic function of $\beta$ in the domain $\operatorname{Re} \beta<0$, and ${ }_{0} D_{R}^{\beta} g_{v}(t)$ defined by (2.4) is its analytic continuation to the whole complex plane. If we assume that $v$ also takes a complex value, ${ }_{0} D_{R}^{\beta} g_{v}(t)$ defined by (2.4) is an analytic function of $v$ in the domain $\operatorname{Re} v>0$. The analytic continuation as a function of $v$ was also studied. The argument is naturally concluded that (2.5) should apply for the analytic continuation, even in $\operatorname{Re} v \leq 0$ except at the points where $v \in \mathbb{Z}_{<1} ;$ see [9].

We now adopt this analytic continuation of ${ }_{0} D_{R}^{\beta} g_{v}(t)$ to represent ${ }_{0} D_{R}^{\beta} g_{v}(t)$, and hence we accept the following lemma.

Lemma 2 (2.5) holds for every $\beta \in \mathbb{R}, \quad v \in \mathbb{R} \backslash \mathbb{Z}_{<1}$.

By (2.1) and (2.5), we have

$$
{ }_{0} D_{R}^{\beta} u(t)=\sum_{\substack{v \in S \\ v-\beta \notin \mathbb{Z}_{<1}}} u_{v-1} g_{v-\beta}(t) .
$$

For $u(t)$ defined by (2.1), we note that

$$
\left[{ }_{0} D_{R}^{-M} u(t)\right] H(t)
$$

is locally integrable on $\mathbb{R}$.

\subsection{Fractional Integral and Derivative of a Distribution}

We consider distributions belonging to $\mathcal{D}_{R}^{\prime}$. When a function $h(t)$ is locally integrable on $\mathbb{R}$ and has a support bounded on the left, it belongs to $\mathcal{D}_{R}^{\prime}$ and is called a regular distribution. The distributions in $\mathcal{D}_{R}^{\prime}$ are called right-sided distributions.

A compact formal definition of a distribution in $\mathcal{D}_{R}^{\prime}$ and its fractional integral and derivative is given in Appendix $\mathbf{A}$ of [4].

Let $f(t) H(t)$ be a regular distribution. Then $\left[{ }_{0} D_{R}^{-\lambda} f(t)\right] H(t)$ for $\lambda \geq 0$ is also a regular distribution, and distribution $D^{-\lambda}[f(t) H(t)]$ is defined by

$$
D^{-\lambda}[f(t) H(t)]=\left[{ }_{0} D_{R}^{-\lambda} f(t)\right] H(t), \lambda \geq 0 .
$$

Let $N \in \mathbb{Z}_{>0}$, and let $h(t)$ be such a regular distribution that $\frac{\mathrm{d}^{n}}{\mathrm{~d} t^{n}} h(t)$ is continuous and differentiable on $\mathbb{R}$, for every $n \in \mathbb{Z}_{[0, N-1]}$. Then $D^{N} h(t)$ is defined by

$$
D^{N} h(t)=\frac{\mathrm{d}^{N}}{\mathrm{~d} t^{N}} h(t) .
$$

Let $\beta \in \mathbb{R}_{>0}, \quad N=\lceil\beta\rceil$, and let

$$
\frac{\mathrm{d}^{n}}{\mathrm{~d} t^{n}}\left[\left\{{ }_{0} D_{R}^{\beta-N} f(t)\right\} H(t)\right]
$$

be continuous and differentiable on $\mathbb{R}$ for every $n \in \mathbb{Z}_{[0, N-1]}$. Then

$$
D^{\beta}[f(t) H(t)]=\left[{ }_{0} D_{R}^{\beta} f(t)\right] H(t) .
$$

When $h(t)$ is a regular distribution, $D^{v} h(t)$ is defined for all $v \in \mathbb{R}$.

Lemma 3 For $h(t) \in \mathcal{D}^{\prime}$, the index law:

$$
D^{\lambda} D^{\beta} h(t)=D^{\lambda+\beta} h(t),
$$

is valid for every $\lambda, \beta \in \mathbb{R}$.

Dirac's delta function $\delta(t)$ is the distribution defined by $\delta(t)=D H(t)$.

Let $\tilde{g}_{v}(t)$ for $v \in \mathbb{R}$ be defined by

$$
\tilde{g}_{v}(t)=D^{-v} \delta(t) .
$$

Lemma 4 If $v \in \mathbb{R}_{>0}$,

$$
\tilde{g}_{v}(t)=g_{v}(t) H(t) .
$$

Proof By putting $f(t)=1$ in (2.7) and using (2.11) and (2.5), we obtain

$$
\begin{aligned}
D^{-v} H(t) & =\left[{ }_{0} D_{R}^{-v} 1\right] H(t) \\
& =g_{v+1}(t) H(t), \quad v \geq 0 .
\end{aligned}
$$

By operating $D$ to this and using (2.9) and (2.5), we obtain (2.12).

Corresponding to $u(t)$ expressed by (2.1), we define $\tilde{u}(t)$ by

$$
\tilde{u}(t)=\sum_{v \in S} u_{v-1} \tilde{g}_{v}(t) .
$$

Then $\tilde{u}(t)$ and $\tilde{f}(t)$ are expressed as

$$
\tilde{u}(t)=\hat{u}(D) \delta(t), \tilde{f}(t)=\hat{f}(D) \delta(t)
$$

where

$$
\hat{u}(D)=\sum_{v \in S} u_{v-1} D^{-v} .
$$

Because of (2.11), we have

$$
D^{\beta} \tilde{g}_{v}(t)= \begin{cases}\tilde{g}_{v-\beta}(t), & v-\beta \in \mathbb{R} \backslash \mathbb{Z}_{<1}, \\ \tilde{g}_{-k}(t)=D^{k} \delta(t), & -k=v-\beta \in \mathbb{Z}_{<1} .\end{cases}
$$

Lemma 5 Let $\beta=-v \in \mathbb{R}$. Then

$$
\begin{aligned}
& t \cdot \tilde{g}_{v}(t)=v \cdot \tilde{g}_{v+1}(t), \\
& t \cdot D^{\beta} \delta(t)=-\beta \cdot D^{\beta-1} \delta(t)=-\frac{\partial}{\partial D} D^{\beta} \delta(t) .
\end{aligned}
$$

The last derivative with respect to $D$ is taken regarding $D$ as a variable.

A proof of (2.17) for $\beta \in \mathbb{R}_{>0}$ is given in Appendix B of [4].

Proof When $\beta=-v, v \in \mathbb{R}_{>0}$, by Lemmas 4 and 1 ,

$$
\begin{aligned}
t \cdot \tilde{g}_{v}(t) & =t \cdot g_{v}(t) H(t) \\
& =v \cdot g_{v+1}(t) H(t) \\
& =v \cdot \tilde{g}_{v+1}(t),
\end{aligned}
$$


The first equality in (2.18) is obtained from (2.17) and vice versa, by using (2.11).

The following lemma is a consequence of this lemma.

Lemma 6 Let $\tilde{u}(t)$ be expressed as a linear combination of $\tilde{g}_{v}(t)$ for $v \in \mathbb{R}$. Then

$$
t \cdot \tilde{u}(t)=t \cdot \hat{u}(D) \delta(t)=-\frac{\partial}{\partial D} \hat{u}(D) \delta(t) .
$$

\subsection{From $u(t)$ to $\tilde{u}(t)$ and Vice Versa}

Lemma 7 Let $v \in \mathbb{R} \backslash \mathbb{Z}_{<1}, \quad N \in \mathbb{Z}_{>-1}$ satisfy $v+N>0$. Then

$$
\begin{aligned}
& \tilde{g}_{v}(t)=D^{N}\left[\left\{{ }_{0} D_{R}^{-N} g_{v}(t)\right\} H(t)\right], \\
& t \cdot \tilde{g}_{v}(t)=D^{N}\left[{ }_{0} D_{R}^{-N}\left\{t \cdot g_{v}(t)\right\} H(t)\right] .
\end{aligned}
$$

Proof Formula (2.20) is derived by applying (2.3), (2.12) and (2.16) to the righthand. Formula (2.21) follows from (2.20) by replacing $g_{v}(t)$ and $\tilde{g}_{v}(t)$ by $t \cdot g_{v}(t)=v \cdot g_{v+1}(t)$, and $t \cdot \tilde{g}_{v}(t)=v \cdot \tilde{g}_{v+1}(t)$, respectively, by using (2.2) and (2.17).

By using Lemma 7 to (2.6), we obtain

$$
\begin{aligned}
& D^{N}\left[{ }_{0} D_{R}^{-N}\left\{{ }_{0} D_{R}^{\beta} u(t)\right\} H(t)\right] \\
= & \sum_{v \in S} u_{v-1} \tilde{g}_{v-\beta}(t) \\
v-\beta \notin \mathbb{Z}_{<1} & D^{N}\left[{ }_{0} D_{R}^{-N}\left\{t \cdot{ }_{0} D_{R}^{\beta} u(t)\right\} H(t)\right] \\
= & t \sum_{v \in S} u_{v-1} \tilde{g}_{v-\beta}(t) \\
v-\beta \notin \mathbb{Z}_{<1} &
\end{aligned}
$$

Lemma 8 Let $v \in \mathbb{R} \backslash \mathbb{Z}_{<1}, N \in \mathbb{Z}_{>-1}$ satisfy $v+N>0$. Then

$$
g_{v}(t) H(t)={ }_{0} D_{R}^{N}\left[D^{-N} \tilde{g}_{v}(t)\right] .
$$

This follows from (2.20).

Condition B $\tilde{u}(t)$ is expressed as a linear combination of $\tilde{g}_{v}(t)$ for $v \in \tilde{S}$, where $\tilde{S}$ is a set of $v \in \mathbb{R}_{>-M} \backslash \mathbb{Z}_{<1}$, for some $M \in \mathbb{Z}_{>-1}$.

When this condition is satisfied, $\tilde{u}(t)$ is expressed as (2.13) with $S$ replaced by $\widetilde{S}$.

Lemma 9 Let $\tilde{u}(t)$ satisfy Condition $B$. Then the corresponding $u(t)$ is obtained from $\tilde{u}(t)$, by

$$
u(t) H(t)={ }_{0} D_{R}^{N}\left[D^{-N} \tilde{u}(t)\right],
$$

and is expressed by (2.1) with $S$ replaced by $\tilde{S}$.

Lemma 10 Let $\tilde{u}(t)$ and $u(t)$ be given by (2.13) and (2.1), respectively. Then $D^{\beta} \tilde{u}(t)$ and $\left\{{ }_{0} D_{R}^{\beta} u(t)\right\} H(t)$ are related by

$$
\begin{aligned}
D^{\beta} \tilde{u}(t) & =D^{N}\left[{ }_{0} D_{R}^{-N}\left\{{ }_{0} D_{R}^{\beta} u(t)\right\} H(t)\right] \\
& +\sum_{\substack{k \in \mathbb{Z}_{>-1} \\
\beta-k \in S}} u_{\beta-k-1} \cdot D^{k} \delta(t)
\end{aligned}
$$

$$
\begin{aligned}
t \cdot D^{\beta} \tilde{u}(t)= & D^{N}\left[{ }_{0} D_{R}^{-N}\left\{t \cdot{ }_{0} D_{R}^{\beta} u(t)\right\} H(t)\right] \\
& +t \sum_{\substack{k \in \mathbb{Z}>-1 \\
\beta-k \in S}} u_{\beta-k-1} \cdot D^{k} \delta(t)
\end{aligned}
$$

if $N \in \mathbb{Z}_{>-1}$ satisfies $N \geq M+\beta$.

Proof By (2.13) and (2.16), we have

$$
\begin{aligned}
D^{\beta} \tilde{u}(t) & =\sum_{\substack{v \in S \\
v-\beta \notin \mathbb{Z}_{<1}}} u_{v-1} \tilde{g}_{v-\beta}(t) \\
& +\sum_{\substack{k \in \mathbb{Z}>-1 \\
\beta-k \in S}} u_{\beta-k-1} \tilde{g}_{-k}(t)
\end{aligned}
$$

Using (2.22) in the first term on the righthand side, we obtain (2.26). Multiplying (2.28) by $t$ and noting that the first term on the righthand side is then equal to (2.23), we obtain (2.27).

\section{Recipe of Solving Laplace's DE and fDE of That Type}

We now express the DE/fDE (1.2) to be solved, as follows:

$$
\sum_{l=0}^{m}\left(a_{l} t+b_{l}\right) \cdot{ }_{0} D_{R}^{l \sigma} u(t)=f(t), \quad t>0,
$$

where $\sigma=\frac{1}{2}$ or $\sigma=1$, and $m=2$. In Sections 4 and 5, we study this DE for $\sigma=1$ and this fDE for $\sigma=\frac{1}{2}$, respectively.

\subsection{Deform to DE/fDE for Distribution}

Using Lemma 10, we express (3.1) as

$$
\sum_{l=0}^{m}\left(a_{l} t+b_{l}\right) \cdot D^{l \sigma} \tilde{u}(t)=\tilde{f}(t)+\tilde{v}(t)
$$

where

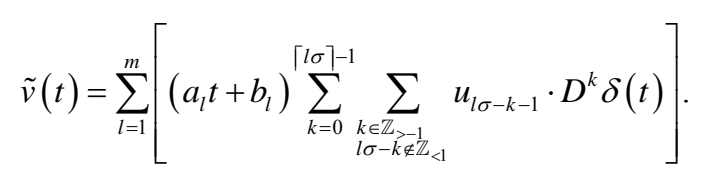

\subsection{Solution Via Operational Calculus}

By using (2.14) and (2.19), we express (3.2) as

$$
\begin{aligned}
& -\sum_{l=0}^{m} a_{l} \cdot \frac{\partial}{\partial D}\left[D^{l \sigma} \hat{u}(D) \delta(t)\right]+\sum_{l=0}^{m} b_{l} \cdot D^{l \sigma} \hat{u}(D) \delta(t) \\
& =-A(D)\left[\frac{\partial}{\partial D} \hat{u}(D) \delta(t)\right]+B(D)[\hat{u}(D) \delta(t)] \\
& =\hat{f}(D) \delta(t)+\hat{v}(D) \delta(t),
\end{aligned}
$$

where 


$$
\begin{aligned}
& A(D)=\sum_{l=0}^{m} a_{l} \cdot D^{l \sigma}, \\
& B(D)=\sum_{l=0}^{m}\left(-l \sigma \cdot a_{l} \cdot D^{l \sigma-1}+b_{l} \cdot D^{l \sigma}\right) \\
& \hat{v}(D)= \\
& \sum_{l=1}^{m}\left[-a_{l} \sum_{\substack{k \in \mathbb{Z}_{>-1} \\
l \sigma-k \notin \mathbb{Z}_{<1}}} k \cdot u_{l \sigma-k-1} \cdot D^{k-1}+b_{l} \sum_{\substack{k \in \mathbb{Z}_{>-1} \\
l \sigma-k \notin \mathbb{Z}_{<1}}} u_{l \sigma-k-1} \cdot D^{k}\right]
\end{aligned}
$$

In order to solve the Equation (3.4) for

$$
\tilde{u}(t)=\hat{u}(D) \delta(t),
$$

we solve the following equation for function $\hat{u}(x)$ of real variable $x$ :

$$
\begin{aligned}
& -A(x) \frac{\mathrm{d}}{\mathrm{d} x} \hat{u}(x)+B(x) \hat{u}(x) \\
& =\hat{f}(x)+\hat{v}(x) .
\end{aligned}
$$

Lemma 11 The complementary solution (C-solution) of equation (3.7) is given by $\hat{u}(x)=C_{1} \cdot \hat{\phi}(x)$, where $C_{1}$ is an arbitrary constant and

$$
\hat{\phi}(x)=C_{2} \cdot \exp \left(\int^{x} \frac{B(\xi)}{A(\xi)} \mathrm{d} \xi\right),
$$

where the integral is the indefinite integral and $C_{2}$ is any constant.

Lemma 12 Let $\hat{\phi}(x)$ be the C-solution of (3.7), and $\hat{u}_{v}^{*}(x)$ be the particular solution (P-solution) of (3.7), when the inhomogeneous term is $x^{-v}$ for $v \in \mathbb{R}$. Then

$$
\hat{u}_{v}^{*}(x)=-\hat{\phi}(x) \int^{x} \frac{\xi^{-v}}{A(\xi) \hat{\phi}(\xi)} \mathrm{d} \xi+C_{3} \cdot \hat{\phi}(x),
$$

where $C_{3}$ is any constant.

Since $f(t) H(t)$ satisfies Condition $A$ and $\hat{v}(D)$ is given by (3.6), the P-solution $\hat{u}(x)$ of (3.7) is expressed as a linear combination of $\hat{u}_{v}^{*}(x)$ for $v \in \mathbb{R}_{>-M}$, and of $\hat{u}_{-k}^{*}(x)$ for $k \in \mathbb{Z}_{>-1}$, respectively.

From the solution $\hat{u}(x)$ of (3.7), $\hat{u}(D)$ is obtained by substituting $x$ by $D$. Then we confirm that (3.4) is satisfied by that $\hat{u}(D)$ operated to $\delta(t)$.

\subsection{Neumann Series Expansion}

Finally the obtained expression of $\hat{u}(D)$ is expanded into Neumann series [11]. Practically we expand it into the sum of terms of negative powers of $D$, and then we obtain the solution $\hat{u}(D) \delta(t)$ of (3.4). If the obtained $\hat{u}(D)$ is a linear combination of $D^{-v}$ for $v \in \mathbb{R}_{>-M}$ with some $M \in \mathbb{Z}_{>-1}$, then $\hat{u}(D) \delta(t)$ is the solution $\tilde{u}(t)$ of (3.2). If it satisfies Condition $\mathrm{B}$, it is converted to a solution $u(t)$ of (3.1) for $t>0$, with the aid of Lemma 9.

\subsection{Recipe of Obtaining the Solution of (3.1)}

1) We prepare the data: $\hat{f}(D)$ by (2.14), and $A(x)$, $B(x)$ and $\hat{v}(D)$ by (3.5) and (3.6).

2) We obtain $\hat{\phi}(x)$ by (3.8). The C-solution of (3.2) is given by

$$
\tilde{u}(t)=C_{1} \cdot \hat{\phi}(D) \delta(t)
$$

If $\tilde{v}(D)=0$, the C-solution of (3.1) is obtained from this with the aid of Lemma 9.

3) If $\hat{f}(D) \neq 0$ or $\hat{v}(D) \neq 0$, we obtain $\hat{u}_{v}^{*}(x)$ given by (3.9).

4) If $\hat{v}(D)=\sum_{k \in \mathbb{Z}_{>-1}} c_{k} D^{k} \neq 0$ and $\tilde{f}(t)=0$, the solution of (3.2) is given by

$$
\tilde{u}(t)=C_{1} \cdot \hat{\phi}(D) \delta(t)+\sum_{k \in \mathbb{Z}_{>-1}} c_{k} \cdot \hat{u}_{-k}^{*}(D) \delta(t),
$$

where $c_{k}$ are constants. The C-solution of (3.1) is then obtained from this with the aid of Lemma 9.

5) If $\hat{f}(D)=\sum_{k=1}^{\infty} d_{k} D^{-v_{k}} \neq 0$, the P-solution of (3.2) is given by

$$
\tilde{u}(t)=\sum_{k=1}^{\infty} d_{k} \cdot \hat{u}_{v_{k}}^{*}(D) \delta(t), t>0,
$$

where $v_{k} \in \mathbb{R}_{>-M}$ and $d_{k}$ are constants. The P-solution of (3.1) with inhomogeneous term

$$
f(t)=\sum_{k=1}^{\infty} d_{k} \cdot g_{c_{k}}(t)
$$

is obtained from this with the aid of Lemma 9.

\subsection{Comments on the Recipe}

In the above recipe, we first obtain the C-solution of (3.7), that is $\hat{u}(x)=C_{1} \cdot \hat{\phi}(x)$. It gives the C-solution $\hat{u}(D) \delta(t)$ of (3.4) and hence the C-solutions $\tilde{u}(t)$ of (3.2). A C-solution $u(t)$ of (3.1) is then obtained with the aid of Lemma 9.

We next obtain the P-solution $\hat{u}_{v}^{*}(x)$ of (3.7), when the inhomogeneous part is $x^{-v}$ for $v \in \mathbb{R}$. As noted above, the P-solutions $\hat{u}(x)$ of (3.7) for $\hat{f}(x)$ and for $\hat{v}(x)$, are expressed as a linear combination of $\hat{u}_{v}^{*}(x)$ for $v \in \mathbb{R}_{>-M}$, and of $\hat{u}_{-k}^{*}(x)$ for $k \in \mathbb{Z}_{>-1}$, respectively. The sum of the P-solutions $\hat{u}(x)$ of (3.7) for $\hat{f}(x)$ and for $\hat{v}(x)$ gives the P-solution $\hat{u}(D) \delta(t)$ of (3.4) and hence the P-solution $\tilde{u}(t)$ of (3.2). The C-solution $u(t)$ of (3.1) comes from the C-solution of (3.7) and the P-solution of (3.7) for $\hat{v}(x)$.

\subsection{Remarks}

When we obtain $\hat{u}(D)$ at the end of Section 3.2, we must examine whether it is compatible with Condition B. We will find that if $b_{l} \neq 0$ for $l \sigma>m \sigma-1$, the obtained $\hat{u}(D)$ is not acceptable. Hence we have to solve 
the problem, assuming that $b_{l}=0$ for all $l>m-1 / \sigma$. When $\sigma=\frac{1}{2}$ and $m=2$, we put $b_{2}=b_{1}=0$. When $\sigma=1$ and $m=2$, we put $b_{2}=0$. Discussion of this problem is given in Appendices C and D of [4]. In the present case, the discussion must be read taking Condition $\mathrm{B}$ there to represent the present Condition B.

\section{Laplace's and Kummer's DE}

We now consider the case of $\sigma=1, m=2, a_{2} \neq 0, a_{1} \neq 0$, and $a_{0}=b_{2}=0$. Then (3.1) reduces to

$$
\begin{aligned}
& a_{2} t \cdot \frac{\mathrm{d}^{2}}{\mathrm{~d} t^{2}} u(t)+\left(a_{1} t+b_{1}\right) \cdot \frac{\mathrm{d}}{\mathrm{d} t} u(t) \\
& +b_{0} \cdot u(t)=f(t), t>0 .
\end{aligned}
$$

By (3.5) and (3.6), $A(x), B(x)$ and $\hat{v}(x)$ are

$$
\begin{aligned}
& A(x)=a_{2} x^{2}+a_{1} x=a_{2} x(x+\alpha), \\
& B(x)=\left(b_{1}-2 a_{2}\right) x+b_{0}-a_{1}, \\
& \hat{v}(x)=\left(-a_{2}+b_{1}\right) u_{0},
\end{aligned}
$$

where $\alpha=\frac{a_{1}}{a_{2}}$.

\subsection{Complementary Solution of (3.7), (3.2) and (4.1)}

In order to obtain the C-solution $\hat{\phi}(x)$ of (3.7) by using (3.8), we express $B(x) / A(x)$ as follows:

$$
\frac{B(x)}{A(x)}=\frac{\gamma_{1}}{x}+\frac{\gamma_{2}}{x+\alpha}
$$

where

$$
\begin{aligned}
& \gamma_{1}+\gamma_{2}=\frac{b_{1}}{a_{2}}-2, \quad \gamma_{1}=\frac{b_{0}}{a_{1}}-1, \\
& \gamma_{2}=\frac{b_{1}}{a_{2}}-\frac{b_{0}}{a_{1}}-1 .
\end{aligned}
$$

$B(x)$ is now expressed as $B(x)=a_{2}\left(\gamma_{1}+\gamma_{2}\right) x+a_{1} \gamma_{1}$.

By using (3.8), we obtain

$$
\begin{aligned}
\hat{\phi}(x) & =x^{\gamma_{1}}(x+\alpha)^{\gamma_{2}} \\
& =x^{\gamma_{1}+\gamma_{2}}\left(1+\alpha x^{-1}\right)^{\gamma_{2}} \\
& =\sum_{n=0}^{\infty}\left(\begin{array}{c}
\gamma_{2} \\
n
\end{array}\right) \alpha^{n} x^{-n+\gamma_{1}+\gamma_{2}},
\end{aligned}
$$

where $\left(\begin{array}{l}\gamma \\ n\end{array}\right)=\frac{(-1)^{n}(-\gamma)_{n}}{n !}$ for $\gamma \in \mathbb{R}$ and $n \in \mathbb{Z}_{>-1}$ are the binomial coefficients.

The C-solution of (3.2) is given by

$$
\begin{aligned}
\tilde{u}(t) & =\hat{u}(D) \delta(t)=C_{1} \cdot \hat{\phi}(D) \delta(t) \\
& =C_{1} \cdot D^{\gamma_{1}+\gamma_{2}}\left(1+\alpha D^{-1}\right)^{\gamma_{2}} \delta(t) \\
& =C_{1} \sum_{n=0}^{\infty}\left(\begin{array}{c}
\gamma_{2} \\
n
\end{array}\right) \alpha^{n} D^{-n+\gamma_{1}+\gamma_{2}} \delta(t) .
\end{aligned}
$$

If $\gamma_{1}+\gamma_{2} \notin \mathbb{Z}_{>-1}$, we obtain a C-solution of (4.1), by using Lemma 9:

$$
\begin{aligned}
& u(t) H(t)=C_{1} \cdot \phi(t) \\
& =C_{1} \sum_{n=0}^{\infty}\left(\begin{array}{c}
\gamma_{2} \\
n
\end{array}\right) \alpha^{n} \frac{1}{\Gamma\left(n-\gamma_{1}-\gamma_{2}\right)} t^{n-\gamma_{1}-\gamma_{2}-1} H(t) \\
& =C_{1} \frac{1}{\Gamma\left(-\gamma_{1}-\gamma_{2}\right)} t^{-\gamma_{1}-\gamma_{2}-1} \\
& \sum_{n=0}^{\infty} \frac{\left(-\gamma_{2}\right)_{n}}{n !\left(-\gamma_{1}-\gamma_{2}\right)_{n}}(-\alpha t)^{n} H(t) \\
& =C_{1} \frac{1}{\Gamma\left(-\gamma_{1}-\gamma_{2}\right)} t^{-\gamma_{1}-\gamma_{2}-1} \\
& { }_{1} F_{1}\left(-\gamma_{2} ;-\gamma_{1}-\gamma_{2} ;-\alpha t\right) H(t) .
\end{aligned}
$$

Remark 1 In Introduction, Kummer's DE is given by (1.4). It is equal to (4.1) for $a_{2}=1, a_{1}=-1, b_{1}=c$ and $b_{0}=-a$. In this case,

$$
\begin{aligned}
& \gamma_{2}=c-a-1, \quad \gamma_{1}+\gamma_{2}=c-2, \\
& \gamma_{1}=a-1, \quad \alpha=-1 .
\end{aligned}
$$

We then confirm that the expression (4.8) for $c \notin \mathbb{Z}_{>1}$ agrees with (1.6), which is one of the C-solutions of Kummer's DE given in $[7,8]$.

\subsection{Particular Solution of (3.7)}

We now obtain the P-solution of (3.7), when the inhomogeneous term is equal to $x^{-v}$ for $v \in \mathbb{R}$.

When the C-solution of (3.7) is $\hat{\phi}(x)$, the P-solution of (3.7) is given by (3.9). By using (4.2) and (4.6), the following result is obtained in [4]:

$$
\begin{aligned}
\hat{u}_{v}^{*}(x) & =-x^{\gamma_{1}}(x+\alpha)^{\gamma_{2}} \int^{x} \frac{\xi^{-v}}{a_{2} \xi^{1+\gamma_{1}}(\xi+\alpha)^{1+\gamma_{2}}} \mathrm{~d} \xi \\
& +C_{3} \cdot \hat{\phi}(x) \\
& =\frac{1}{a_{2}} \sum_{n=0}^{\infty}{ }_{n} C_{\gamma_{2}, \gamma_{1}+1+v}^{*} \cdot \alpha^{n} x^{-n-1-v},
\end{aligned}
$$

where

$$
{ }_{n} C_{p_{1}, p_{2}}^{*}=\sum_{k=0}^{n}\left(\begin{array}{c}
p_{1} \\
k
\end{array}\right)\left(\begin{array}{c}
-p_{1}-1 \\
n-k
\end{array}\right) \frac{1}{n-k+p_{1}+p_{2}} .
$$

Lemma 13 When $p_{1}+p_{2} \notin \mathbb{Z}_{<1},{ }_{n} C_{p_{1}, p_{2}}^{*}$ defined by (4.11) is expressed as 


$$
\begin{aligned}
{ }_{n} C_{p_{1}, p_{2}}^{*} & =\frac{(-1)^{n}}{p_{1}+p_{2}} \cdot{ }_{n} C_{p_{1}, p_{2}}^{\dagger}, \\
{ }_{n} C_{p_{1}, p_{2}}^{\dagger} & =\frac{\left(p_{2}\right)_{n}}{\left(1+p_{1}+p_{2}\right)_{n}} .
\end{aligned}
$$

This lemma is proved in [4].

\subsection{Particular Solutions of (3.2) and (4.1)}

Equation (4.10) shows that if the inhomogeneous term is $D^{-v} \delta(t)$ for $v \in \mathbb{R}$, the P-solution of (3.2) is given by

$$
\tilde{u}_{v}^{*}(t)=\frac{1}{a_{2}} \sum_{n=0}^{\infty}{ }_{n} C_{\gamma_{2}, \gamma_{1}+1+v}^{*} \cdot \alpha^{n} D^{-n-1-v} \delta(t) .
$$

Theorem 1 Let $v \in \mathbb{R} \backslash \mathbb{Z}_{<0}, \quad 1+v+\gamma_{1}+\gamma_{2} \notin \mathbb{Z}_{<1}$, and $f(t)=g_{v}(t)$. Then we have a P-solution $u_{v}^{*}(t)$ of (4.1), given by

$$
u_{v}^{*}(t) H(t)=\frac{1}{a_{2} \cdot\left(1+v+\gamma_{1}+\gamma_{2}\right)} u_{v}^{\dagger}(t) H(t),
$$

where

$$
\begin{aligned}
& u_{v}^{\dagger}(t) H(t) \\
& =t^{\nu} \sum_{n=0}^{\infty} \frac{\left(v+1+\gamma_{1}\right)_{n}}{\left(2+v+\gamma_{1}+\gamma_{2}\right)_{n} \Gamma(v+n+1)}(-\alpha t)^{n} H(t) .
\end{aligned}
$$

Proof Applying Lemma 9 to (4.13), we obtain

$$
\begin{aligned}
& u_{v}^{*}(t) H(t) \\
& =\frac{1}{a_{2}} \sum_{n=0}^{\infty}{ }_{n} C_{\gamma_{2}, \gamma_{1}+1+v}^{*} \frac{1}{\Gamma(n+1+v)} \alpha^{n} \cdot t^{n+v} H(t) .
\end{aligned}
$$

By using (4.12) in (4.16), we obtain (4.14) with (4.15).

We note that $u_{v}^{\dagger}(t) H(t)$ is expressed as

$$
\begin{aligned}
& u_{v}^{\dagger}(t) H(t)=\frac{1}{\Gamma(v+1)} t^{v} \\
& \sum_{n=0}^{\infty} \frac{\left(v+1+\gamma_{1}\right)_{n}(1)_{n}}{\left(2+v+\gamma_{1}+\gamma_{2}\right)_{n}(v+1)_{n} n !}(-\alpha t)^{n} H(t) \\
= & \frac{1}{\Gamma(v+1)} t^{v} \\
{ }_{2} & F_{2}\left(v+1+\gamma_{1}, 1 ; 2+v+\gamma_{1}+\gamma_{2}, v+1 ;-\alpha t\right) H(t) .
\end{aligned}
$$

\subsection{Complementary Solution of (4.1)}

By (4.3) and (4.5), $\hat{v}(x)=a_{2} \cdot\left(\gamma_{1}+\gamma_{2}+1\right) u_{0}$. When $\hat{f}(x)=0$ and $\hat{v}(x) \neq 0$, the P-solution of (4.7) is given by

$$
\hat{u}(x)=a_{2} \cdot\left(\gamma_{1}+\gamma_{2}+1\right) \cdot u_{0} \cdot \hat{u}_{0}^{*}(x) .
$$

By using (4.14) for $v=0$, if $1+\gamma_{1}+\gamma_{2} \notin \mathbb{Z}_{<1}$, we obtain a C-solution of (4.1):

$$
\begin{aligned}
u(t) H(t) & =u_{0} \cdot u_{0}^{\dagger}(t) H(t) \\
& =u_{0} \sum_{n=0}^{\infty} \frac{\left(1+\gamma_{1}\right)_{n}}{\left(2+\gamma_{1}+\gamma_{2}\right)_{n} n !}(-\alpha t)^{n} H(t) \\
& =u_{0} \cdot{ }_{1} F_{1}\left(1+\gamma_{1} ; 2+\gamma_{1}+\gamma_{2} ;-\alpha t\right) H(t) .
\end{aligned}
$$

In Section 4.1, we have (4.8), that is another C-solution of (4.1). If we compare (4.8) with (4.15), when $\gamma_{1}+\gamma_{2} \notin \mathbb{Z}_{>-1}$, it can be expressed as

$$
C_{1} \cdot \phi(t) H(t)=C_{1} \cdot u_{-\gamma_{1}-\gamma_{2}-1}^{\dagger}(t) H(t) .
$$

Proposition 1 When $\gamma_{1}+\gamma_{2} \notin \mathbb{Z}$, the complementary solution of (4.1), multiplied by $H(t)$, is given by the sum of the righthand sides of (4.8) and of (4.20), which are equal to $C_{1} \cdot u_{-\gamma_{1}-\gamma_{2}-1}^{\dagger}(t) H(t)$ and $u_{0} \cdot u_{0}^{\dagger}(t) H(t)$, respectively.

Remark 2 As stated in Remark 1, for Kummer's DE, $\gamma_{1}$ and $\gamma_{2}$ are given in (4.9), and

$$
1+\gamma_{1}=a, \quad 2+\gamma_{1}+\gamma_{2}=c, \quad \alpha=-1 .
$$

We then confirm that if $c \notin \mathbb{Z}$, the set of (4.8) and (4.20) agrees with the set of (4.5) and (4.6).

\subsection{Remarks}

In [10], it was shown that there exist P-solutions expressed by a polynomial for inhomogeneous Hermite's $\mathrm{DE}$, et al. We can obtain the corresponding result for Laplace's DE. We discuss this problem in Appendix A, and then discuss the P-solution of inhomogeneous Hermite's DE in the present formulation in Appendix B.

\section{Solution of fDE (3.1) for $\sigma=1 / 2$}

In this section, we consider the case of $\sigma=\frac{1}{2}, m=2$, $a_{2} \neq 0, a_{1} \neq 0, b_{0} \neq 0$, and $a_{0}=b_{2}=b_{1}=0$,

Then the Equation (3.1) to be solved is

$$
\begin{aligned}
& a_{2} t \cdot{ }_{0} D_{R} u(t)+a_{1} t \cdot{ }_{0} D_{R}^{1 / 2} u(t)+b_{0} \cdot u(t) \\
& =f(t), t>0 .
\end{aligned}
$$

Now (3.5) and (3.6) are expressed as

$$
\begin{aligned}
& A(x)=a_{2} x+a_{1} x^{1 / 2}=a_{2} x^{1 / 2}\left(x^{1 / 2}+\alpha\right), \\
& B(x)=b_{0}-a_{2}-\frac{1}{2} a_{1} x^{-1 / 2}, \\
& \hat{v}(x)=-a_{1} \sum_{k=0}^{M}(k+1) u_{1 / 2-k-2} \cdot x^{k},
\end{aligned}
$$

where $\alpha=\frac{a_{1}}{a_{2}}$. 


\subsection{Complementary Solution of (3.7)}

By using (5.2), $B(x) / A(x)$ is expressed as

$$
\frac{B(x)}{A(x)}=x^{-1 / 2}\left(\frac{\gamma_{1}}{x^{1 / 2}}+\frac{\gamma_{2}}{x^{1 / 2}+\alpha}\right)
$$

where

$$
\gamma_{1}+\gamma_{2}=\frac{b_{0}}{a_{2}}-1, \quad \gamma_{1}=-\frac{1}{2}, \quad \gamma_{2}=\frac{b_{0}}{a_{2}}-\frac{1}{2} .
$$

By (3.8), the C-solution $\hat{\phi}(x)$ of (3.7) is given by

$$
\begin{aligned}
\hat{\phi}(x) & =x^{\gamma_{1}}\left(x^{1 / 2}+\alpha\right)^{2 \gamma_{2}}=x^{\gamma_{1}+\gamma_{2}}\left(1+\alpha x^{-1 / 2}\right)^{2 \gamma_{2}} \\
& =\sum_{n=0}^{\infty}\left(\begin{array}{c}
2 \gamma_{2} \\
n
\end{array}\right) \alpha^{n} x^{-n / 2+\gamma_{1}+\gamma_{2}} .
\end{aligned}
$$

\subsection{Complementary Solution of (3.2) and (5.1)}

The C-solution of (3.2) is given by

$$
\begin{aligned}
\tilde{u}(t) & =\hat{u}(D) \delta(t)=C_{1} \cdot \hat{\phi}(D) \delta(t) \\
& =C_{1} \sum_{n=0}^{\infty}\left(\begin{array}{c}
2 \gamma_{2} \\
n
\end{array}\right) \alpha^{n} D^{-n / 2+\gamma_{1}+\gamma_{2}} \delta(t) .
\end{aligned}
$$

If $2 \gamma_{1}+2 \gamma_{2} \notin \mathbb{Z}_{>0}$, by applying Lemma 9 to this, we obtain the C-solution of (5.1):

$$
\begin{aligned}
& u(t) H(t)=C_{1} \cdot \phi(t) H(t) \\
& =C_{1} \sum_{n=0}^{\infty}\left(\begin{array}{c}
2 \gamma_{2} \\
n
\end{array}\right) \frac{1}{\Gamma\left(\frac{n}{2}-\gamma_{1}-\gamma_{2}\right)} \alpha^{n} t^{n / 2-\gamma_{1}-\gamma_{2}-1} H(t) .
\end{aligned}
$$

\subsection{Particular Solution of (3.2) and (5.1)}

By using the expressions of $A(x)$ and $\hat{\phi}(x)$ given by (5.2) and (5.6) in (3.9), we obtain the P-solution of (3.7), when the inhomogeneous term is $x^{-v}$ for $v \in \mathbb{R}$ :

$$
\begin{aligned}
\hat{u}_{v}^{*}(x) & =-x^{\gamma_{1}}\left(x^{1 / 2}+\alpha\right)^{2 \gamma_{2}} \\
& \cdot \int^{x} \frac{\xi^{-v}}{a_{2} \xi^{1 / 2+\gamma_{1}}\left(\xi^{1 / 2}+\alpha\right)^{1+2 \gamma_{2}}} \mathrm{~d} \xi+C_{3} \cdot \hat{\phi}(x) \\
& =\frac{2}{a_{2}} \sum_{n=0}^{\infty}{ }_{n} C_{2 \gamma_{2}, 2 \gamma_{1}+2 v}^{*} \cdot \alpha^{n} x^{-n / 2-v},
\end{aligned}
$$

where ${ }_{n} C_{2 \gamma_{2}, 2 \gamma_{1}+2 v}^{*}$ is defined by (4.11) and is given by (4.12), if $2 v+2 \gamma_{1}+2 \gamma_{2} \notin \mathbb{Z}_{<1}$.

By using (4.12) in (5.9), we can show that if the inhomogeneous term is $D^{-v} \delta(t)$ for $v \in \mathbb{R}$, the P-solution of (3.2) is $\tilde{u}_{v}^{*}(t)=\hat{u}_{v}^{*}(D) \delta(t)$. By applying Lemma 9 to this, we obtain the following theorem.

Theorem 2 Let $v \in \mathbb{R}, 2 v \notin \mathbb{Z}_{<1}, 2 v+2 \gamma_{1}+2 \gamma_{2} \notin \mathbb{Z}_{<1}$, and $f(t)=\frac{1}{\Gamma(v)} t^{v-1}$. Then we have a P-solution $u_{v}^{*}(t)$ of (5.1), given by

$$
u_{v}^{*}(t) H(t)=\frac{1}{a_{2} \cdot\left(v+\gamma_{1}+\gamma_{2}\right)} u_{v}^{\dagger}(t) H(t),
$$

where

$$
\begin{aligned}
u_{v}^{\dagger}(t) H(t)= & \sum_{n=0}^{\infty} \frac{\left(2 v+2 \gamma_{1}\right)_{n}}{\left(1+2 v+2 \gamma_{1}+2 \gamma_{2}\right)_{n}} \\
& \cdot \frac{1}{\Gamma\left(\frac{n}{2}+v\right)}(-\alpha)^{n} t^{n / 2+v-1} H(t) .
\end{aligned}
$$

In Appendix C, discussion is given to show that there exist P-solutions in the form of polynomial for (5.1).

\subsection{Complementary Solution of (5.1)}

We obtain the solution $u_{v}^{*}(t)$ only for $v \notin \mathbb{Z}_{<1}$. Even though we have P-solutions of (3.2) for $\tilde{v}(t)=\hat{v}(D) \delta(t)$, when $\hat{v}(x)$ is given by (5.3) with nonzero values of $u_{1 / 2-k-2}$, it does not satisfy Condition $\mathrm{B}$, and does not give a solution of (5.1). Hence $u(t)$ given by (5.8) is the only C-solution of (5.1).

If we compare (5.8) with (5.11), we obtain the following proposition.

Proposition 2 Let $2 \gamma_{1}+2 \gamma_{2} \notin \mathbb{Z}_{>0}$. Then the C-solution of (5.1) is given by

$$
C_{1} \cdot \phi(t) H(t)=C_{1} \cdot u_{-\gamma_{1}-\gamma_{2}}^{\dagger}(t) H(t)
$$

\section{REFERENCES}

[1] K. Yosida, "The Algebraic Derivative and Laplace's Differential Equation," Proceedings of the Japan Academy, Vol. 59, Ser. A, 1983, pp. 1-4.

[2] K. Yosida, "Operational Calculus," Springer-Verlag, New York, 1982, Chapter VII.

[3] J. Mikusiński, "Operational Calculus," Pergamon Press, London, 1959.

[4] T. Morita and K. Sato, "Remarks on the Solution of Laplace's Differential Equation and Fractional Differential Equation of That Type," Applied Mathematics, Vol. 4, No. 11A, 2013, pp. 13-21.

[5] T. Morita and K. Sato, "Solution of Fractional Differential Equation in Terms of Distribution Theory," Interdisciplinary Information Sciences, Vol. 12, No. 2, 2006, pp. 71-83.

[6] T. Morita and K. Sato, "Neumann-Series Solution of Fractional Differential Equation," Interdisciplinary Information Sciences, Vol. 16, No. 1, 2010, pp. 127-137.

[7] M. Abramowitz and I. A. Stegun, "Handbook of Mathematical Functions with Formulas, Graphs and Mathematical Tables," Dover Publ., Inc., New York, 1972, Chapter 13.

[8] M. Magnus and F. Oberhettinger, "Formulas and Theorems for the Functions of Mathematical Physics," Chelsea 
Publ. Co., New York, 1949, Chapter VI.

[9] T. Morita and K. Sato, "Liouville and Riemann-Liouville Fractional Derivatives via Contour Integrals," Fractional Calculus and Applied Analysis, Vol. 16, No. 3, 2013, pp. 630-653.

[10] L. Levine and R. Maleh, "Polynomial Solutions of the
Classical Equations of Hermite, Legendre and Chebyshev," International Journal of Mathematical Education in Science and Technology, Vol. 34, 2003, pp. 95-103.

[11] F. Riesz and B. Sz.-Nagy, "Functional Analysis," Dover Publ., Inc., New York, 1990, p. 146. 


\section{Appendix A: Polynomial Form of P-Solution of (4.1)}

Let $v_{1} \in \mathbb{R} \backslash \mathbb{Z}_{<0}$ and $n_{1} \in \mathbb{Z}_{>0}$. Then (4.15) gives

$$
\begin{aligned}
u_{v_{1}+n_{1}}^{\dagger}(t) H(t) & =\frac{1}{(-\alpha)^{n_{1}} \cdot{ }_{n_{1}} C_{\gamma_{2}, \gamma_{1}+1+v_{1}}^{\dagger}} t^{\nu_{1}} \sum_{n=n_{1}}^{\infty}{ }_{n} C_{\gamma_{2}, \gamma_{1}+1+v_{1}}^{\dagger} \frac{1}{\Gamma\left(v_{1}+n+1\right)}(-\alpha t)^{n} H(t) \\
& =\frac{1}{(-\alpha)^{n_{1}} \cdot{ }_{n_{1}} C_{\gamma_{2}, \gamma_{1}+1+v_{1}}^{\dagger}}\left[u_{v_{1}}^{\dagger}(t)-t^{\nu_{1}} \sum_{n=0}^{n_{1}-1}{ }_{n} C_{\gamma_{2}, \gamma_{1}+1+v_{1}}^{\dagger} \frac{1}{\Gamma\left(v_{1}+n+1\right)}(-\alpha t)^{n}\right] H(t)
\end{aligned}
$$

where

$$
{ }_{n} C_{\gamma_{2}, \gamma_{1}+1+v_{1}}^{\dagger}=\frac{\left(v_{1}+1+\gamma_{1}\right)_{n}}{\left(2+v_{1}+\gamma_{1}+\gamma_{2}\right)_{n}} .
$$

We obtain the following theorems from (A.2) with the aid of Proposition 1.

Theorem 3 Let $n_{1} \in \mathbb{Z}_{>0}, \quad \gamma_{1} \notin \mathbb{Z}_{<0}, \gamma_{1}+\gamma_{2} \notin \mathbb{Z}_{<0}$, and $f(t)=g_{n_{1}}(t)$. Then we have the polynomial form of $P$-solution of (4.1):

$$
u(t) H(t)=-\frac{1}{a_{2} \cdot\left(1+n_{1}+\gamma_{1}+\gamma_{2}\right)} \cdot \frac{\left(2+\gamma_{1}+\gamma_{2}\right)_{n_{1}}}{\left(1+\gamma_{1}\right)_{n_{1}}(-\alpha)^{n_{1}}} \sum_{n=0}^{n_{1}-1} \frac{\left(1+\gamma_{1}\right)_{n}}{\left(2+\gamma_{1}+\gamma_{2}\right)_{n} \Gamma(n+1)}(-\alpha t)^{n} H(t) .
$$

Theorem 4 Let $n_{1} \in \mathbb{Z}_{>0}, \quad \gamma_{1}+\gamma_{2} \notin \mathbb{Z}_{>-1}, \quad \gamma_{2} \notin \mathbb{Z}_{>-1}$ and $f(t)=g_{v}(t)$ for $v=-\gamma_{1}-\gamma_{2}-1+n_{1}$. Then we have the polynomial form of $P$-solution of (4.1):

$$
u(t) H(t)=-\frac{1}{a_{2} n_{1}} \cdot \frac{(1)_{n_{1}}}{\left(-\gamma_{2}\right)_{n_{1}}(-\alpha)^{n_{1}}} t^{-\gamma_{1}-\gamma_{2}-1} \sum_{n=0}^{n_{1}-1} \frac{\left(-\gamma_{2}\right)_{n}}{(1)_{n} \Gamma\left(-\gamma_{1}-\gamma_{2}+n\right)}(-\alpha t)^{n} H(t) .
$$

\section{Appendix B: Polynomial Form of P-Solution of Hermite DE}

We now consider the inhomogeneous Hermite DE given by

$$
\frac{\mathrm{d}^{2}}{\mathrm{~d} x^{2}} y(x)-2 x \frac{\mathrm{d}}{\mathrm{d} x} y(x)+2 c \cdot y(x)=x^{\mu},
$$

for $x \in \mathbb{R}$ and $\mu \in \mathbb{R}_{>-1}$. We put $t=x^{2}$ and $u(t)=y(x)$. Then the equation for $u(t)$ is given by

$$
4 t \frac{\mathrm{d}^{2}}{\mathrm{~d} t^{2}} u(t)+(-4 t+2) \frac{\mathrm{d}}{\mathrm{d} t} u(t)+2 c \cdot u(t)=t^{\mu / 2}
$$

This is Laplace's DE (4.1) with parameters

$$
\begin{aligned}
& a_{2}=4, a_{1}=-4, b_{1}=2, b_{0}=2 c, \gamma_{1}+\gamma_{2}=-\frac{3}{2}, \\
& \gamma_{1}=-\frac{c}{2}-1, \gamma_{2}=\frac{c}{2}-\frac{1}{2},
\end{aligned}
$$

and the inhomogeneous term $f(t)=\Gamma\left(\frac{\mu}{2}+1\right) g_{\mu / 2+1}(t)$.

Theorem 5 Let $c=2 m+1, m \in \mathbb{Z}$, and $\mu=2\left(n_{1}-1\right)$, $n_{1} \in \mathbb{Z}_{>0}$. Then we have the polynomial form of $P$ solution of (B.2):

$$
u(t) H(t)=-\frac{\left(\frac{1}{2}\right)_{n_{1}} \Gamma\left(n_{1}\right)}{4\left(n_{1}-\frac{1}{2}\right)\left(-\frac{1}{2}-m\right)_{n_{1}}} \sum_{n=0}^{n_{1}-1} \frac{\left(-\frac{1}{2}-m\right)_{n}}{\left(\frac{1}{2}\right)_{n} \Gamma(n+1)} t^{n} H(t) .
$$

Proof In this case, $\gamma_{1}=-m-\frac{3}{2}, \gamma_{2}=m$, and $n_{1}=\frac{\mu}{2}+1$. By Theorem 3, we obtain this result.
Theorem 6 Let $c=2 m+1, m \in \mathbb{Z}_{<0}$, and $\mu=2 n_{1}-1$, $n_{1} \in \mathbb{Z}_{>0}$. Then we have the polynomial form of $P$ solution of (B.2): 


$$
u(t) H(t)=-\frac{(1)_{n_{1}} \Gamma\left(\frac{1}{2}+n_{1}\right)}{4 n_{1}(-m)_{n_{1}}} t^{1 / 2} \sum_{n=0}^{n_{1}-1} \frac{(-m)_{n}}{(1)_{n} \Gamma\left(n+\frac{3}{2}\right)} t^{n} H(t) .
$$

Proof In this case, $\gamma_{1}=-m-\frac{3}{2}, \gamma_{2}=m$, and $\frac{1}{2}+n_{1}=\frac{\mu}{2}+1$. By Theorem 4 , we obtain this result.

$$
u(t) H(t)=-\frac{(1)_{n_{1}} \Gamma\left(\frac{1}{2}+n_{1}\right)}{4 n_{1}\left(\frac{1}{2}-m\right)_{n_{1}}} t^{1 / 2} \sum_{n=0}^{n_{1}-1} \frac{\left(\frac{1}{2}-m\right)_{n}}{(1)_{n} \Gamma\left(\frac{3}{2}+n\right)} t^{n} H(t) .
$$

Proof In this case, $\gamma_{1}=-m-1, \quad \gamma_{2}=m-\frac{1}{2}$, and $\frac{1}{2}+n_{1}=\frac{\mu}{2}+1$. By Theorem 4 , we obtain this result.
Theorem 7 Let $c=2 m, m \in \mathbb{Z}$, and $\mu=2 n_{1}-1$, $n_{1} \in \mathbb{Z}_{>0}$. Then we have the polynomial form of $P$ solution of (B.2):

$$
u(t) H(t)=-\frac{\left(\frac{1}{2}\right)_{n_{1}} \Gamma\left(n_{1}\right)}{4\left(n_{1}-\frac{1}{2}\right)(-m)_{n_{1}}} \sum_{n=0}^{n_{1}-1} \frac{(-m)_{n}}{\left(\frac{1}{2}\right)_{n} \Gamma(n+1)} t^{n} H(t) .
$$

Proof In this case, $\gamma_{1}=-m-1, \quad \gamma_{2}=m-\frac{1}{2}$, and $n_{1}=\frac{\mu}{2}+1$. By Theorem 3, we obtain this result.

Remark 3 We confirm that Theorems 7 and 5, res-
Theorem 8 Let $c=2 m, m \in \mathbb{Z}_{<0}$, and $\mu=2 n_{1}-2$, $n_{1} \in \mathbb{Z}_{>0}$. Then we have the polynomial form of $P$ solution of (B.2):

$$
\begin{aligned}
u_{v_{1}+n_{1}}^{\dagger}(t) H(t) & =\frac{1}{(-\alpha)^{n_{1}} \cdot{ }_{n_{1}} C_{2 \gamma_{2}, 2 \gamma_{1}+2 v_{1}}^{\dagger}} t^{\nu_{1}-1} \sum_{n=n_{1}}^{\infty} C_{2 \gamma_{2}, 2 \gamma_{1}+2 v_{1}}^{\dagger} \frac{1}{\Gamma\left(v_{1}+\frac{n}{2}\right)}(-\alpha)^{n} t^{n / 2} H(t) \\
& =\frac{1}{(-\alpha)^{n_{1}} \cdot{ }_{n_{1}} C_{2 \gamma_{2}, 2 \gamma_{1}+2 v_{1}}^{\dagger}}\left[u_{v_{1}}^{\dagger}(t)-t^{\nu_{1}-1} \sum_{n=0}^{n_{1}-1}{ }_{n} C_{2 \gamma_{2}, 2 \gamma_{1}+2 v_{1}}^{\dagger} \frac{1}{\Gamma\left(v_{1}+\frac{n}{2}\right)}(-\alpha)^{n} t^{n / 2}\right] H(t),
\end{aligned}
$$

where

$$
{ }_{n} C_{2 \gamma_{2}, 2 \gamma_{1}+2 v_{1}}^{\dagger}=\frac{\left(2 v_{1}+2 \gamma_{1}\right)_{n}}{\left(1+2 v_{1}+2 \gamma_{1}+2 \gamma_{2}\right)_{n}} .
$$

We obtain the following theorem from (C.2) with the aid of Proposition 2.

Theorem 9 Let $n_{1} \in \mathbb{Z}_{>0}, \quad \gamma_{1}+\gamma_{2} \notin \mathbb{Z}_{>-1}, \quad \gamma_{2} \notin \mathbb{Z}_{>-1}$ and $f(t)=g_{v}(t)$ for $v=-\gamma_{1}-\gamma_{2}+n_{1}$. Then we have the polynomial form of $P$-solution of (5.1):

$$
u(t) H(t)=-\frac{1}{a_{2} n_{1}} \cdot \frac{(1)_{n_{1}}}{\left(-2 \gamma_{2}\right)_{n_{1}}(-\alpha)^{n_{1}}} t^{-\gamma_{1}-\gamma_{2}-1} \sum_{n=0}^{n_{1}-1} \frac{\left(-2 \gamma_{2}\right)_{n}}{(1)_{n} \Gamma\left(-\gamma_{1}-\gamma_{2}+\frac{n}{2}\right)}(-\alpha)^{n} t^{n / 2} H(t) .
$$

